Contemporary Mathematics

\title{
Some more non-arithmetic rigid groups
}

\author{
Alexander Lubotzky \\ Dedicated to the memory of Bob Brooks
}

\section{Introduction}

Let $\Gamma$ be a finitely generated group. $\Gamma$ is called rigid if for every $n \geq 1, \Gamma$ admits only finitely many isomorphism classes of irreducible representations into $G L_{n}(\mathbf{C})$. Platonov (cf. [PR p. 437) conjectured that if $\Gamma$ is a linear rigid group then $\Gamma$ is of "arithmetic type" (i.e., commensurable with a product of $S$-arithmetic groups).

In $\mathbf{B L}, \mathrm{H}$. Bass and the author gave counter-examples to Platonov's conjecture. The examples $\Gamma$ there are very special; they are subgroups of $L \times L$ when $L$ is a uniform (arithmetic) lattice in the rank one simple Lie group $F_{4}^{(-20)}$. The proof there relies on four main ingredients:

(1) The super-rigidity (á la Margulis) of $L$, which was proved by Corlette $\mathbf{C}$ and Gromov-Schoen [GS.

(2) The Ol'shanskiu-Rips theorem $([\mathrm{O}])$ asserting that $L$ being hyperbolic has a finitely presented infinite quotient $H$ with no proper finite index subgroup.

(3) Grothendieck's theorem [GO] which says that once the inclusion of $\Gamma$ to $L \times L$ induces an isomorphism of the pro-finite completions $\hat{\Gamma}$ and $\hat{L} \times \hat{L}$, the representation theory of $\Gamma$ is the same as that of $L \times L$.

(4) The vanishing of the second Betti number $\beta_{2}\left(L^{\prime}\right)=\operatorname{dim} H^{2}\left(L^{\prime}, \mathbf{R}\right)=0$ for every finite index subgroup $L^{\prime}$ of $L$ (proved by Kumareson and VoganZuckerman (cf. $[\mathbf{V Z}]$ )).

In this note we give a simplified version of the proof in $\mathbf{B L}$. We eliminate the use of ingredients (3) and (4). Not using (3) makes the proof more elementary, but avoiding (4) is even more significant: our proof now works also when $L$ is any uniform lattice in $S p(n, 1)$ (for every $n \geq 2$ ), groups for which (4) does not hold (as follows from ( $[\mathbf{L i}]$, Cor. 6.5). We thus have many new examples which are Zariski dense in $S p(n, 1) \times S p(n, 1)$.

2000 Mathematics Subject Classification. 22E40.

The author is indebted to the Israel Science Foundation and the U.S.-Israel Binational Science Foundation for their support.

(C) 0000 (copyright holder) 
Just as in $\mathbf{B L}$, the counter examples to Platonov's conjecture constructed here are even super-rigid (see $\S 1$ ). In summary:

Theorem. Let $G$ be either $S p(n, 1), n \geq 2$, or $F_{4}^{-(20)}$ and let $L$ be a cocompact lattice in $G$. Then $L \times L$ contains a subgroup $\Gamma$ of infinite index which is Zariski dense in $G \times G$, super-rigid and not of arithmetic type.

We end the note with two suggestive remarks to be explained in $\S 4$ :

We show that if $L$ satisfies the congruence subgroup property, then a further simplification is possible, avoiding also the use of (1) and (2), i.e. the works of Corlette, Gromov-Schoen, Ol'shanskii and Rips.

Furthermore, if a single uniform lattice $L$ in $S p(n, 1)$ or $F_{4}^{(-20)}$ satisfies the congruence subgroup property, then there exists an hyperbolic group á la Gromov which is not residually-finite. Moreover, there exists a hyperbolic group with no proper finite index subgroup. So, answering the congruence subgroup problem in the affirmative, for one such $L$ would settle in negative the long standing open problem on the residual-finiteness of hyperbolic groups.

\section{FAb fibre products of rigid groups are rigid}

Throughout the paper, $\Gamma$ is a finitely generated group. $\Gamma$ is FAb if for every finite index subgroup $\Lambda$ of $\Gamma, \Lambda^{a b}:=\Lambda /[\Lambda, \Lambda]$ is finite. $\Gamma$ is rigid if for every $n \geq 1$, it has only finitely many non-equivalent irreducible $n$-dimensional complex representations. Finally, $\Gamma$ is super-rigid if $A(\Gamma)^{0}$, the connected component of $A(\Gamma)$ is finite-dimensional algebraic group. Here, $A(\Gamma)$ is the pro-algebraic completion of $\Gamma$. There is a homomorphism $i: \Gamma \rightarrow A(\Gamma)$ such that for every representation $\rho: \Gamma \rightarrow G L_{n}(\mathbf{C})$, there exists a unique algebraic representation $\tilde{\rho}: A(\Gamma) \rightarrow G L_{n}(\mathbf{C})$ with $\tilde{\rho} \circ i=\rho$.

It is not difficult to prove:

Proposition 1.1. super-rigid $\Rightarrow$ rigid $\Rightarrow F A b$.

Proposition 1.2. If $\Lambda$ is a finite index subgroup of $\Gamma$, then $\Lambda$ is super-rigid (resp. rigid, $F A b)$ iff $\Gamma$ is.

We also have:

Proposition 1.3. Assume $\Gamma$ has FAb. Then given $n$, there exists a finite index subgroup $\Lambda=\Lambda(n)$ of $\Gamma$ such that for every $n$-dimensional representation

$\rho: \Gamma \rightarrow G L_{n}(\mathbf{C}), \overline{\rho(\Lambda)}$ is a connected group. If $\rho$ is irreducible then $\overline{\rho(\Lambda)}$ is semisimple.

Proof. Denote $H=\overline{\rho(\Gamma)}$, then there exists a finite subgroup $F$ of $H$ such that $H=H^{0} \cdot F$ ( $\mathbf{W}$ 10.10). By Jordan's Theorem ( $\mathbf{W}$ 9.2), $F$ has an abelian normal subgroup of index at most $J(n)$. Thus the same applies to $H / H^{0}$. Let now $\tilde{\Lambda}$ be the intersection of all the normal subgroups of $\Gamma$ of index at most $J(n)$ and $\Lambda=[\tilde{\Lambda}, \tilde{\Lambda}]$. It follows that $\Lambda$ has finite index in $\Gamma$ and for every $n$-dimensional representation of $\Gamma, \rho(\Lambda) \subseteq \overline{\rho(\Gamma)}^{0}$. This also implies that $\rho(\Lambda)$ is dense in $\overline{\rho(\Gamma)}^{0}$ since $\Lambda$ has finite index in $\Gamma$ and $\overline{\rho(\Gamma)}^{0}$ has no finite index subgroups. Finally, if $\rho$ is irreducible, then $\overline{\rho(\Gamma)}^{0}$ is reductive, but actually semisimple as it has no abelian quotient. 
Proposition 1.4. A finitely generated FAb group $\Gamma$ is not rigid iff there exists a finite index subgroup $\Lambda$ of $\Gamma$ and a simple algebraic group $H$ of adjoint type (i.e., $Z(H)=1$ ) such that $\Lambda$ has infinitely many non-conjugate homomorphisms $\rho: \Lambda \rightarrow H$ with $\rho(H)$ Zariski dense in $H$.

Proof. Assume there are infinitely many non-equivalent irreducible $n$-dimensional representations $\rho$ of $\Gamma$ and let $\Lambda=\Lambda(n)$ as in Proposition 1.3. Then $\overline{\rho(\Lambda)}$ is a connected semisimple group for every such $\rho$. There are only finitely many conjugacy classes of semisimple connected subgroups of $G L_{n}(\mathbf{C})$, so we may assume that $H=\overline{\rho(\Lambda)}$ is fixed. From Clifford theorem [W, Theorem 1.7], it follows that there are infinitely many non-equivalent $\Lambda$-representations with Zariski-dense image in $H$. Infinitely many of them are still non-equivalent when $H$ is divided by its finite center. We can therefore assume $H$ is of adjoint type and a direct product of its simple components. There are still infinitely many non-equivalent homomorphisms of $\Lambda$ to one of the simple factors of $H$. Replacing $H$ by this factor gives the result. The other direction is easy as $(\operatorname{Aut}(H): \operatorname{Inn}(H))$ is finite.

We now show how new rigid (resp. super-rigid) groups can be obtained as a fibre product of rigid (resp. super-rigid) groups.

For $i=1,2$, let $L_{i}$ be a finitely generated group with epimorphisms $\rho_{i}$ from $L_{i}$ onto the same finitely presented group $D$, and $R_{i}=\operatorname{Ker} \rho_{i}$. Let

$$
\Gamma=L_{1} \times{ }_{D} L_{2}=\left\{(x, y) \in L_{1} \times L_{2} \mid \rho_{1}(x)=\rho_{2}(y)\right\}
$$

be the fibre product of $L_{1}$ and $L_{2}$ over $D$.

The projections $\pi_{i}$ of $\Gamma$ to $L_{i}$ are onto with kernels $\left(1, R_{2}\right)$ and $\left(R_{1}, 1\right)$. Also, one can easily see that $\Gamma$ is finitely generalized since $D$ is finitely presented.

Proposition 1.5. If $L_{1}$ and $L_{2}$ are rigid and $\Gamma$ has $F A b$, then $\Gamma$ is also rigid.

Proof. If not, by Proposition 1.4, there exists a finite index subgroup $\Lambda$ of $\Gamma$ and a simple algebraic group $H$ that $\Lambda$ has infinitely many non-equivalent homo-

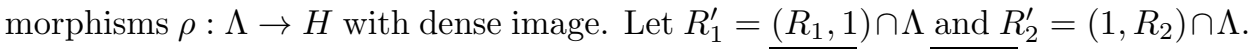
Then $\left[R_{1}^{\prime}, R_{2}^{\prime}\right]=1$ and both are normal in $\Lambda$. Thus $\overline{\rho\left(R_{1}^{\prime}\right)}$ and $\overline{\rho\left(R_{2}^{\prime}\right)}$ are commuting normal subgroups of $H$, so one of them must be trivial. Hence for infinitely many $\rho$ 's, either $\rho\left(R_{1}^{\prime}\right)$ is trivial or $\rho\left(R_{2}^{\prime}\right)$ is. In the first case we have that $\rho$ factors through $\pi_{2}$, i.e., we have a representation of $\Lambda / R_{1}^{\prime}=\Lambda /\left(R_{1}, 1\right) \cap \Lambda \simeq \Lambda\left(R_{1}, 1\right) /\left(R_{1}, 1\right)$ which is commensurable to $L_{2}$. Hence, this group is not rigid and by Proposition 1.2 also $L_{2}$ is not rigid, a contradiction. The case with $\rho\left(R_{2}^{\prime}\right)=1$ is treated similarly and the Proposition is proved.

It is somewhat more difficult to prove, but under an additional hypothesis, it is also true that when we start with $L_{i}$ super-rigid and reductive, then $\Gamma$ is super-rigid.

Recall that a group is called reductive if every representation of it is reductive.

Proposition 1.6. Assume $L_{1}$ and $L_{2}$ are super-rigid and reductive, $D$ has no finite index subgroup and $\Gamma$ is FAb then $\Gamma$ is super-rigid and reductive, in fact $A(\Gamma)^{0}=A\left(L_{1}\right)^{0} \times A\left(L_{2}\right)^{0}$

Proof. Let $\rho: \Gamma \rightarrow G L_{n}(\mathbf{C})$ be a representation. Replacing $\Gamma$ by $\Lambda$ as in Proposition 1.3, we can assume $H=\overline{\rho(\Lambda)}$ is connected and it is equal to its own commutator subgroup (since $\Gamma$ is FAb). 
We claim that $H$ is semi-simple. If not, it has a non-trivial unipotent radical $U$. Dividing by $[U, U]$ and further if needed, we can assume that $U$ is a simple $H$-module. So $H=U \cdot S$, a semi-direct product when $S$ is semisimple and $U$ a simple $S$-module. Clearly, this is not the trivial module, since $H$ has no abelian quotient.

As in the proof of (1.5), $\overline{\rho\left(R_{1}^{\prime}\right)}$ and $\overline{\rho\left(R_{2}^{\prime}\right)}$ are commuting normal subgroups. Moreover, $\Lambda / R_{1}^{\prime} \times R_{2}^{\prime} \simeq D$ and $D$ has no finite index subgroups, hence no finite dimensional representations. It follows that $\overline{\rho\left(R_{1}^{\prime} \times R_{2}^{\prime}\right)}=\overline{\rho\left(R_{1}^{\prime}\right)} \cdot \overline{\rho\left(R_{2}^{\prime}\right)}=H$.

If $\overline{\rho\left(R_{1}^{\prime}\right)}$ does not contain $U$, then $H / \overline{\rho\left(R_{1}^{\prime}\right)}$ has non-trivial unipotent radical and so $\Lambda / R_{1}^{\prime}$, which is commensurable to $L_{2}$ is not reductive, a contradiction. Hence $\overline{\rho\left(R_{1}^{\prime}\right)}$, and similarly $\overline{\rho\left(R_{2}^{\prime}\right)}$, contains $U$. But $\left[\overline{\rho\left(R_{1}\right)}, \overline{\rho\left(R_{2}^{\prime}\right)}\right]=1$, since $\left[R_{1}^{\prime}, R_{2}^{\prime}\right]=1$. This implies that $\left[U, \overline{\rho\left(R_{2}^{\prime}\right)}\right]=\left[\overline{\rho\left(R_{1}^{\prime}\right)}, U\right]=1$ and hence $H$ acts trivially on $U$, a contradiction. Hence $U$ is trivial and $H$ is semisimple. Again $\overline{\rho\left(R_{1}^{\prime}\right)}$ and $\overline{\rho\left(R_{2}^{\prime}\right)}$ are normal and commuting. This implies that (after dividing by the center of $H$ ) $H=\overline{\rho\left(R_{1}^{\prime}\right)} \times \overline{\rho\left(R_{2}^{\prime}\right)}$ but then $H / \overline{\rho\left(R_{i}^{\prime}\right)}$ is an homomorphic image of $A\left(L_{3-i}\right)^{0}$. This proves that $A(\Gamma)^{0}=A\left(L_{1}\right)^{0} \times A\left(L_{2}\right)^{0}$.

\section{When a fibre product is FAb}

In this section we show some sufficient conditions for a fibre product to be FAb.

Let $L$ be a finitely generated group, $\rho: L \rightarrow D$ a homomorphism onto a finitely presented group $D$ with kernel $R$, and $\Gamma=L \times_{D} L$ the fibre product of $L$ over $D$,

$$
\Gamma=\{(x, y) \in L \times L \mid \rho(x)=\rho(y)\} .
$$

Note that $\Gamma=(R, 1) \Delta(L)=(1, R) \Delta(L)$ when $\Delta(L)$ is the diagonal embedding of $L$ into $\Gamma, \Delta(L)=\{(x, x) \mid x \in L\}$.

Lemma 2.1. If $L$ is $F A b$ and if for every finite index normal subgroup $L_{0}$ of $L$, and every finite index subgroup $R_{0}$ of $R$ which is normal in $L,\left[L_{0}, R_{0}\right]$ is of finite index in $R_{0}$, then $\Gamma$ is FAb.

Proof. Let $\Gamma_{0}$ be a finite index normal subgroup of $\Gamma, L_{0}=\Gamma_{0} \cap \Delta(L)$ and $R_{0}=(R, 1) \cap \Gamma_{0}$. By abuse of notation we will consider $R_{0}$ as a subgroup of $L$ and at the same time a subgroup of $(L, 1)$. ¿From our assumption, it follows that $\left[R_{0}, L_{0}\right]$ is of finite index in $R_{0}$ and hence in $(R, 1)$. As $L$ is FAb, $\left[L_{0}, L_{0}\right]$ is of finite index in $\Delta(L)$. This implies that $\left[R_{0} L_{0}, R_{0} L_{0}\right]$ is of finite index in $\Gamma$. As $\Gamma_{0} \supseteq R_{0} L_{0}$, we get that $\left[\Gamma_{0}, \Gamma_{0}\right]$ is of finite index in $\Gamma$.

Let now $L$ be a finitely generated group with FAb. Assume $L$ has an infinite finitely presented quotient $D$ with kernel $R$, such that $\hat{D}=\{1\}$ when $\hat{D}$ is the profinite completion of $D$, i.e., $D$ has no proper finite index subgroup. Clearly $D^{a b}=\{1\}$, i.e, $D$ is a perfect group. It has therefore a universal central extension

$$
1 \rightarrow H_{2}(D) \rightarrow \bar{D} \rightarrow D \rightarrow 1
$$

(cf. $[\mathrm{M}] 55$ ).

Lemma 2.2. Let

$$
1 \rightarrow C \rightarrow E \rightarrow D \rightarrow 1
$$

be a central extension of $D$ such that $E^{a b}$ is finite. Then $r k(C) \leq r k\left(H_{2}(D)\right)$. (For an abelian group $A$ we denote $r k(A)=\operatorname{dim}_{\mathbf{Q}}(A \otimes \mathbf{z} \mathbf{Q})$ ). 
Proof. There is a homomorphism $\psi$ from the universal central extension $(*)$ to (**). We claim that $\psi(\bar{D})$ is of finite index in $E$. Indeed, $C \psi(\bar{D})=E$ and $C$ is central. Hence $\psi(\bar{D}) \triangleleft E$ and the quotient is abelian. But $E^{a b}$ is finite, hence $(E: \psi(\bar{D}))<\infty$. This implies that $\psi\left(H_{2}(D)\right)$ is of finite index in $C$ and hence $r k(C) \leq r k\left(H_{2}(D)\right)$.

Lemma 2.3. For a finite index normal subgroup $L_{0}$ of $L$ denote $R_{0}=R \cap L_{0}$. Then $r k\left(R_{0} /\left[L_{0}, R_{0}\right]\right) \leq r k\left(H_{2}(D)\right)$ for every $L_{0} \triangleleft L$ of finite index.

Proof. The map $L_{0} \rightarrow D$ is onto since $\hat{D}=1$ and so:

$$
1 \rightarrow R_{0} /\left[L_{0}, R_{0}\right] \rightarrow L_{0} /\left[L_{0}, R_{0}\right] \rightarrow D \rightarrow 1
$$

is a central extension of $D$. As $L$ is FAb, $L_{0}^{a b}$ is finite and our Lemma follows from Lemma 2.2 .

Given $L$ and $\rho: L \rightarrow D$ with $R=\operatorname{Ker} \rho$ as before, choose now $L_{0}$ of finite index with $R_{0}=R \cap L_{0}$ and $r k\left(R_{0} /\left[L_{0}, R_{0}\right]\right)$ maximal among all possible such $L_{0}$. As $\hat{D}=\{1\}$, it follows that $L_{0} / R_{0} \simeq D$ and we have a central extension

$$
1 \rightarrow R_{0} /\left[L_{0}, R_{0}\right] \rightarrow D_{0}:=L_{0} /\left[L_{0}, R_{0}\right] \rightarrow D=L_{0} / R_{0} \rightarrow 1
$$

Now, $R_{0} /\left[L_{0}, R_{0}\right]$ is dense in the profinite topology of $D_{0}$ since $\hat{D}=\{1\}$, hence $\hat{D}_{0}$ is abelian. But, $L_{0}$ is FAb, so $\hat{D}_{0}$ is finite. Replace $L_{0}$ by a finite index subgroup $L_{0}^{\prime}$ so that $D_{1}=L_{0}^{\prime} /\left(\left[L_{0}, R_{0}\right] \cap L_{0}^{\prime}\right)$ satisfies $\hat{D}_{1}=\{1\}$ and in particular $D_{1}^{a b}=\{1\}$. Let us now rename and call $L:=L_{0}^{\prime}, \tilde{D}:=D_{1}$ and $\tilde{R}=\operatorname{Ker}(L \rightarrow \tilde{D})$. So $\tilde{D}$ is a central (possibly infinite!) extension of the original $D$ and $\hat{\tilde{D}}=\{1\}$. We get the exact sequence:

$$
1 \rightarrow \tilde{R} \rightarrow L \rightarrow \tilde{D} \rightarrow 1
$$

The crucial point is:

Claim: For every normal subgroup $L_{1}$ of finite index in $L$ and every $R_{1}$ of finite index in $\tilde{R}$ which is normal in $L,\left[L_{1}, R_{1}\right]$ is of finite index in $R_{1}$.

Proof. The group $\tilde{R} /\left[L_{1}, R_{1}\right]$ is a finitely generated virtually abelian group. Hence $L /\left[L_{1}, R_{1}\right]$ is an extension of the form $1 \rightarrow C \rightarrow L /\left[L_{1}, R_{1}\right] \rightarrow D \rightarrow 1$ where $C$ is virtually abelian and $\hat{D}=\{1\}$. Moreover, $L$ is FAb. So, all this implies that $\left(L / \widehat{\left[L_{1}, R_{1}\right]}\right)$ is finite. Hence, whenever $L /\left[L_{1}, R_{1}\right]$ is mapped into a residually-finite group, its image is finite. This applies, in particular, to the image of $L /\left[L_{1}, R_{1}\right]$ in its action (by conjugation) on the finitely generated virtually abelian group $\tilde{R} /\left[L_{1}, R_{1}\right]$. Thus for some finite index subgroup $L_{2}$ of $L_{1},\left[L_{2}, \tilde{R}\right] \subseteq\left[L_{1}, R_{1}\right]$. By the maximality choice of $L_{0}$, it follows that $\left[L_{2}, \tilde{R}\right]$ is of finite index in $\tilde{R}$ and hence $\left[L_{1}, R_{1}\right]$ is also of finite index there.

To summarize, by replacing $D$ by $\tilde{D}$ (and the original $L$ by a subgroup of finite index), we get an exact sequence:

$$
1 \rightarrow \tilde{R} \rightarrow L \rightarrow \tilde{D} \rightarrow 1
$$

which satisfies the claim. Hence by Lemma 2.1, we have:

Corollary 2.4. The fibre product $\Gamma=L \times_{\tilde{D}} L$ is FAb. 


\section{The main result}

In this section, we pick the fruits of the preparations in the previous two sections and construct non-arithmetic super-rigid groups.

Propositions 1.5 and 1.6 show how to get new (super) rigid groups from old ones. The standard examples of rigid groups are irreducible lattices in semisimple groups of higher rank, but these do not have infinite quotients, so fibre products of them are commensurable to other arithmetic groups. This leads us to lattices in

$S p(n, 1)$ and $F_{4}^{(-20)}$. They are super-rigid $(\mathbf{C}, \mathbf{G S})$ and at the same time have many infinite quotients.

So, from now on in this section, let $L$ be a torsion-free uniform (=cocompact) lattice in one of the groups $S p(n, 1)$ or $F_{4}^{(-20)}$. It is a hyperbolic group and hence by a result proved independently by Ol'shanskii and Rips (see $\mathbf{O}$ ), it has a finitely presented infinite quotient $\rho: L \rightarrow D$ where $D$ has no proper finite index subgroups. Replace $D$ now by $\tilde{D}$ (and $L$ by a finite index subgroup, also called $L$ ) as in $\S 2$. Let $\tilde{\rho}: L \rightarrow \tilde{D}$ be the new map and $\Gamma=L \times_{\tilde{D}} L$ the fibre product. (The reader may note that at this point our method differs from the one in $\mathbf{B L}$, where $L \times_{D} L$ was used).

Theorem 3.1. $\Gamma$ is a super-rigid group. In fact, $A(\Gamma)^{0}=A(L)^{0} \times A(L)^{0}$.

Proof. By Corollary 2.4, $\Gamma$ is FAb. Hence Proposition 1.6 can be applied to deduce that $\Gamma$ is super-rigid (and hence rigid) and $A(\Gamma)^{0}=A(L)^{0} \times A(L)$.

The proof actually shows that every representation of $\Gamma$ can be extended, on a finite index subgroup, to a representation of $L \times L$. One can now repeat the standard argument given in ( $\mathbf{B L}$, pp. 1171-1172) to show that $\Gamma$ is not (virtually) isomorphic to any lattice in a product of linear algebraic groups (over archimedean or non-archimedean fields). So $\Gamma$ is not "of arithmetic type" giving the desired counter-example to Platonov's conjecture.

\section{Remarks on the congruence subgroup property}

Let $L$ be a uniform lattice in $S p(n, 1)$ or $F_{4}^{(-20)}$ as in section 3. Such an $L$ is an arithmetic lattice (GS ). The question whether $L$ satisfies the congruence subgroup property (CSP, for short) is still open. Serre's conjecture, posed in $\mathbf{S}$, suggests that lattices in rank one groups do not have CSP, while lattices in higher rank simple groups do. So by this conjecture, $L$ is not expected to have the CSP. But this conjecture was made in 1970. Since then, it has been shown that in spite of $S p(n, 1)$ and $F_{4}^{(-20)}$ being rank one groups, lattices in them behave in many ways (property $T$, super-rigidity, arithmeticity, etc.) like higher rank lattices. One may, therefore, suggest that they do have the CSP. As of now, the answer is not known for any single such $L$.

We make here two short remarks showing that an affirmative answer for the CSP for $L$ would have two interesting corollaries:

Remark 4.1. If $L$ has the CSP, then the above construction (in $\S 3$ ) of $D$ (and hence of $\Gamma$ ) can be done without appealing to the work of Ol'shanskii and Rips. In fact, if $N$ is any infinite normal subgroup of $L$ (which is finitely generated as normal subgroup) of infinite index, then $D=L / N$ has only finitely many finite index subgroups. Indeed, if not, it has infinitely many finite index normal subgroups. 
Pulling them back to $L$, we get infinitely many normal congruence subgroups of $L$ all containing $N$. But it is not difficult to prove that for $L$ being an arithmetic subgroup of a simple algebraic group, the intersection of any infinite collection of normal congruence subgroups must be finite and central. This shows that $D=$ $L / N$ has only finitely many finite index subgroups. We can replace $D$ by their intersection (and $L$ by the preimage) to get the desired finitely presented quotient without any finite index subgroup.

It is of interest to recall that the CSP implies super-rigidity. Hence, if $L$ has CSP, we can produce a counter-example without appealing to the work of $\mathbf{C}$ and GS. So the proof would not use any of the four ingredients (1)-(4) listed in the introduction.

Remark 4.2. If there is one such uniform lattice $L$ satisfying CSP, then there exists a hyperbolic group without any finite index subgroup and in particular, a non-residually-finite hyperbolic group.

Indeed, $L$ is hyperbolic (but residually finite). For "many" $g \in L$, the normal closure $N$ of $g$ in $L$ is an infinite subgroup of infinite index and $D=L / N$ is also hyperbolic (see $\mathbf{D}$ ). Repeating the argument from remark 4.1, we deduce that $D$ has a finite index subgroup (hence also hyperbolic) with no finite index subgroup. Thus answering the congruence subgroup problem in the affirmative for one uniform

lattice in $S p(n, 1)$ or $F_{4}^{(-20)}$, would solve the long standing problem of the existence of a non-residually finite hyperbolic group.

\section{References}

[BL] H. Bass and A. Lubotzky, Nonarithmetic superrigid groups: counterexamples to Platonov's conjecture, Ann. of Math. 151 (2000), 1151-1173.

[C] K. Corlette, Archimedean superrigidity and hyperbolic geometry, Ann. of Math. 135 (1992), 165-182.

[D] T. Delzant, Sous-groupes distingueés et quotients des groupes hyperboliques, Duke Math J. 83 (1996), 661-682.

[GS] M. Gromov and R. Schoen, Harmonic maps into singular spaces and p-adic superrigidity for lattices in groups of rank one, Inst. Hautes Études Sci. Publ. Math. 76 (1992), 165-246.

[GO] A. Grothendieck, Représentations linéaires et compactification profinie des groupes discrets, Manuscripta Math. 2 (1970), 375-396.

[Li] Li, Jian-Shu, Nonvanishing theorems for the cohomology of certain arithmetic quotients, J. Reine Angew. Math. 428 (1992), 177-217.

[M] J. Milnor, Introduction to Algebraic-K-Theory, Princeton University Press, Princeton, NJ 1971.

[O] Ol'shanskiı̌, A. Yu., On the Bass-Lubotzky question about quotients of hyperbolic groups, J. Algebra 226 (2000), 807-817.

[PR] V. Platonov and A. Rapinchuk, Algebraic Groups and Number Theory, Pure and Applied Mathematics, Academic Press Inc., Boston, MA (1994), xii+614.

[S] J.-P. Serre, Le problème des groupes de congruence pour SL2, Ann. of Math 92 (1970), 489-527.

[VZ] D.A. Vogan Jr. and G.J. Zuckerman, Unitary representations with nonzero cohomology, Compositio Math. 53 (1984), 51-90.

[W] B.A.F. Wehrfritz, Infinite Linear Groups, Springer Berlin, 1973.

Institute of Mathematics, Hebrew University, Jerusalem 91904, ISRAEL

E-mail address: alexlub@math.huji.ac.il 